\title{
Comparative Evaluation of the Diagnostic Performance Characteristics of a One-Step Urine Malaria Test (UMT) against Rapid Diagnostic Tests (RDT) in Febrile Patients from Fako Division, Cameroon
}

Nforbugwe Achu Che Awah ( $\square$ niawah95@gmail.com )

University of Buea Faculty of Health Sciences https://orcid.org/0000-0002-9403-8522

Rengerline Bihnwi Nchotu

University of Buea Faculty of Health Sciences

Agnes Djema Bongah

University of Buea Faculty of Health Sciences

Jules Clement Nguedia Assob

University of Buea Faculty of Health Sciences

Research

Keywords: Malaria, Plasmodium, mosquito

Posted Date: June 10th, 2020

DOI: https://doi.org/10.21203/rs.3.rs-33603/v1

License: (9) This work is licensed under a Creative Commons Attribution 4.0 International License.

Read Full License 


\section{INTRODUCTION}

The disease, Malaria is caused by the Plasmodium species, which is transmitted to humans by the bite of an infected female Anopheles mosquito. These different Plasmodium species have different clinical implications and infect humans in different combinations around the world. Despite the interventions put in place by the World Health Organization to fight Malaria, it still remains a public health priority especially in sub-Saharan Africa [1]. The 2014 World Health Organization report stated that there were about 584,000 malaria deaths annually worldwide, with $78 \%$ of these deaths occurring in children under 5 years old, this largely (>90\%) occurred in Sub-Saharan Africa [2].

Reliable diagnosis of malaria requires laboratory confirmation of the presence of malaria parasites in the blood of a febrile patient. In eastern Africa, where $90 \%$ of the malaria is due to Plasmodium falciparum, accuracy of malaria diagnosis at the outpatient level is becoming increasingly important due to emerging drug resistance and the use of alternative, costly antimalarial drugs [3, 4]. It is estimated that accurate diagnostic tests for malaria have the potential to prevent 400 million unnecessary treatment cases, save 100, 000 lives per year, waste of already scarce resources and impacts positively on the prompt treatment of malaria [5].

Current malaria diagnostic methods necessitate the use of blood for diagnosis, using either RDT and/or microscopy. Although these methods for malaria diagnosis have been reported to be more sensitive, accurate, relatively cheaper and rapid, their greatest disadvantage is their dependency on blood samples for diagnosis. These techniques therefore, introduce the risk of accidental infections from diseases such as Hepatitis B, Malaria, HIV and other blood related diseases which are common in malaria-endemic areas [6, 7]. These techniques also necessitate rigorous training and biological safety precautions, so as to ensure proper containerization and disposal of used needles. The use of needles has also been reported to also serve as a limitation for malaria diagnosis in certain African communities which still regard blood withdrawal as a taboo [7].

The UMT, is a recombinant monoclonal antibody and an immunochromatographic lateral flow assay, that detects $P$. falciparum specific Histidine-Rich Protein 2, a poly-histidine protein or fragments shedded in the urine of febrile patients. HRP2 is produced by merozoite and gametocyte forms of the malaria parasite. The blood then transports HRP2 to the kidneys, where it is passed on to the bladder as part of the urine. The collection of urine is non-invasive, simple, safe, stress free, painless, and can be done by individuals with limited training, including patients. No special equipment is needed for collection and it allows for multiple or serial collections outside of the hospital [8].

There is therefore a need for the development of a non-invasive, simple, rapid, easy to perform, and reliable diagnostic methods, for the prompt and accurate diagnosis of malaria. Hence, the objective of this study was to compare the diagnostic characteristics of the novel UMT to the currently used Blood RDT in an attempt to validate the use of UMT as a diagnostic tool for malaria in the South West Region of Cameroon. The study was also aimed at finding out the efficacy of UMT in detecting low parasitemia in the study population.

\section{METHODOLOGY}

Buea is the capital of the Southwest Region of Cameroon and is located in the eastern slopes of Mount Cameroon. Limbe is the divisional capital of Fako, which host a number of touristic sites and is located at the foot of Mount Cameroon which is about $870 \mathrm{~km}$ above sea level. This study area has two seasons - the dry season (between October and March), and the rainy season (between April and September). Human malaria can be described as mesoendemic in the dry season and hyperendemic in the rainy season, with 
peaks at the beginning and towards the end of the rainy season [9]. The population in this study area experiences an estimated 3.93 infective bites person/night and it has been reported that $P$. falciparum accounts for up to $96 \%$ of malaria infections in this area $[10,11]$. The Buea and Limbe Regional Hospitals provide care to over 10,000 patients annually. Participants were patients who were visiting these hospitals for consultation during this study period.

\section{Study design}

It was a cross sectional comparational study that was conducted in the Limbe and Buea Regional Hospitals from April to August 2017. Structured questionnaires were given to each participant and interviews were done for those who could not read or write. Samples (urine and blood) were collected once from patients who accepted to take part in the study after giving their consents.

\section{Sample population}

A sample size of 200 was used. Those who participated in this study were: febrile patients of either sex regardless of their ages with axillary temperatures $>37.5^{\circ} \mathrm{C}$ or with a history of fever in the previous 48 hours. Pregnant women also participated if they gave their consent. After initial urine screening using the CYBOW $^{\mathrm{TM}}$ Urinalysis test strips, participants with a hematuria, and/or leucocytes $>15 / \mu 1$ and/or urobilinogens $\geq 1 \mathrm{mg} / \mathrm{dl}$ were excluded from the study since these parameters are features of many kidney diseases with probable high levels of antibody that may falsify the study results. Futhermore, those who were presenting with signs and symptoms of Rheumatoid Arthritis (painful joints, inflamed joints, limitation in motion, malaise, and tenderness of the joints) were also not considered since Rheumatoid Arthritis has been proven to give False Positive results for RDT. Finally patients who were already on antimalarial drug were not part of the study.

\section{Sampling technique}

The systematic random sampling technique was used, with daily attendance as sampling frame. Participants were recruited between April and August 2017, which are spanning periods of high malaria transmission in the study area. Recruitment of participants was done daily from Mondays to Fridays in the Limbe and Buea Regional Hospitals. Data was collected from the administration of structured questionnaire and interviews. Furthermore, each participant was given an identification number so as to respect confidentiality.

\section{Laboratory analysis}

\section{Specimen collection}

The main specimens collected were blood and urine. Capillary blood was collected by finger pricking. About $50 \mu 1$ of blood was used to make a thin and thick blood film following standards procedures. Blood was analysed for malaria by malaria RDT and microscopy. Urine was collected in a leak-proofed container for UMT analysis.

\section{Microscopic Examination and quantification of parasites}

The prepared blood films were air-dried and stained with $10 \%$ Giemsa (1 in 20 dilutions) for 25 - 30 min [12]. Two trained and experienced microscopists who did not have prior knowledge of the patients' clinical history, read the slides independently and an average parasitaemia density was obtained. Slides were 
considered positive only when asexual parasite forms - trophozoites and schizonts (not gametocytes alone) - were detected, since asexual forms are indicative of active infection. Parasite densities were determined by using the formula below [13]. A blood film was assumed negative when the examination of 100 thick film fields did not show the presence of asexual forms of $P$. falciparum. Parasitaemia was categorized as low (<1000parasites/ $\mu$ l), moderate (1000-4999parasites/ $\mu$ l blood) and high ( $>5000$ parasites $/ \mu$ l blood).

$$
\text { Parasitaemia per microlitre }=\text { number of parasites } \times 8,000
$$

$$
200 \text { leucocytes. }
$$

\section{Rapid Diagnostic Test (RDT).}

A commercially available RDT kit ( CareStart $^{\mathrm{TM}}$ Malaria HRP2) Combo, ACCESSBIO, INC., New Jersey, USA) was used to detect malaria parasites, according to the manufacturer's instructions, using $5 \mu 1$ of capillary blood. The membrane strips were read and interpreted after $20 \mathrm{~min}$ as either positive, negative or indeterminate [14].

\section{Urine Malaria Test}

A commercially available urine diagnostic test, UMT strip (Fyodor Biotechnologies, Inc. Baltimore MD USA, catalog number UMT-5, Urine Malaria Test ${ }^{\mathrm{TM}} \mathrm{Kit}$ ), ) was also used to diagnose the malaria infection following the manufacturer's instructions. The results were then carefully interpreted as positive, negative or indeterminate [15].

\section{Ethical consideration}

The research protocol was read and approved by the Faculty of Health Sciences. Ethical clearance for this study was obtained from the University of Buea, FHS-Institutional Review Board (2017/023/UB/SG/IRB/FHS). Administrative clearance was obtained from the Regional Delegation of public health for the South West Region. Administrative authorizations were obtained from the Limbe and Buea Regional Hospitals. An informed consent form was made stating the special measures involved to ensure no harm was caused to the participants. The risks and benefits were well stated in this form. The consents of these participants were obtained by signing the consent and accent forms.

\section{Data Management and Statistical Analysis.}

Data was entered into Microsoft excel 2010 software program and double checked for errors before being exported to SPSS version 22 (IBM Inc). Frequency tables were used to present demographic characteristics. Measures of descriptive statistics were used to compute means, median and standard deviation. For determination of sensitivity, specificity, PPV, NPV, PLR, NLR, a $2 \times 2$ cross tabulation of each UMT and RDT against microscopy was done. ROC curves were plotted to evaluate the accuracy of UMT and RDT against microscopy as the gold standard. Linear regression analysis was done to determine the relationship between age and parasite load. The detection limit was calculated from the sample with the lowest parasitaemia having a true positive result. Statistical significance was considered at $\mathrm{P}<0.05$.

\section{RESULTS}

Demographics and clinical characteristics of participants.

Out of a total of 786 individuals who were screened, 200 who met the inclusion criteria were enrolled. In this study, $54.5 \%$ (109) of the participants were females and $45.5 \%$ (91) were males. The mean age was 27 years with range of 9 months to 86 years. The highest malaria prevalence (23.5\%) was seen in the 21-30 age group while the least (6.5\%) was found in the 41-50 age group and the 61-70 age group. All the 
participants presented with fever $\left(\geq 37.5^{\circ} \mathrm{C}\right)$ on enrollment, with a mean body temperature of $38.2^{\circ} \mathrm{C}$. Headaches, body pains, nausea and chills were the most commonly reported symptoms. The participants presented with other diseases like: diabetes, hypertension, typhoid, HIV/AIDS, TB, gastritis and asthma. In this study, $77 \%$ of the population used the Insecticide Treated Mosquito bed-nets while 23\% did not. The distribution of malaria prevalence in the study population is represented in table 1 below. The participants were screened for malaria parasites using Giemsa Microscopy (GM). Among the participants, 93 were positive for $P$. falciparum malaria, giving an overall prevalence of $46.5 \%$. No significant association was observed between the prevalence of malaria and sex $(P=0.345)$. Likewise, no significant association was observed between the prevalence of malaria and age $(P=0.216)$ (see Table 1$)$.

Out of the 93 samples that were positive on GM, 75 were also found positive by the UMT while 74 were found to be positive for RDT. Out of the 107 samples that were negative, as determined by Giemsa Microscopy, 91 were found negative by the UMT while 93 were found Negative for RDT. (see Table 2). The two malaria diagnostic test methods that were evaluated against GM, displayed close similarities in their diagnostic performance characteristics, taking into considerations their specificity, sensitivity, PPV, NPV,PLR and NPV as shown on Table 3.

The lowest parasite density detected was 140 parasites/ $\mu 1$. The proportion of the participants who did not have detectable parasites in their blood was 53.5\% (107 out of the 200 participants who were sampled). Most of the participants who were positive for malaria had parasite density $>200$ parasite/ $\mu 1$. Meanwhile the least proportion of the study population had parasite density of <100parasite/ $\mu 1$. The highest parasite density (57,560parasite/ $\mu 1)$ was seen in the 21-30 age group.

\section{DISCUSSION}

This study demonstrated an analytical sensitivity (AS), the least detectable number of parasites, of UMT to be 140 parasites/ $\mu 1$ which was similar to that of RDT. This detection limit was high compared to other methods such as Microscopy (50 parasites $/ \mu \mathrm{L})$ and PCR $(10-50$ parasites $/ \mu \mathrm{L})$ [16]. The UMT had the lowest limit of detection of 140 parasites $/ \mu 1$, and a $40 \%$ sensitivity at $\leq 200$ parasites/ $\mu 1$. This was similar to a work done by Tagbo Oguonu et al, in 2014 who had as lowest limit of detection of 120 parasites/ $\mu$ l, and a $50 \%$ sensitivity at $\leq 200$ parasites/ $\mu 1$ [17]. Many reasons can be suggested for the relative poor sensitivity at lower parasitaemia levels, which may be related to parasite antigen production, antigen content in urine, cross reactivity with other antibodies in patients and time of urine void. Nwakanma et al. noted that the amount of malaria antigen was low in urine and dependent probably on the time of collection of the samples. They suggested that first void morning urine might probably give better sensitivity than later timed samples [18]. This may not be practicable in clinical practice where the results are required for immediate treatment. With the probable variability in malaria antigen quantity, it is likely that the expected amount of antibody impregnated in the urine-specific test kits as well as the quantity of body fluid required may be higher than those of blood-specific test kits thus necessitating a probable further optimization of the Fyodor UMT to enhance test sensitivity in low parasitaemia. It is known that the property of the antibody impregnated in the nitrocellulose pad of the immunochromatographic test kits also determine the sensitivity. Immunochromatographic tests in which $\operatorname{IgG}$ antibody is used as the coating antibody to capture HRP-2 antigen are likely to give higher rates of false positivity than a test system in which IgM antibody is coated onto the strips [8].

In this study, there was a close similarity in the sensitivity (84.09\% and $82.41 \%)$ and specificity $(83.03 \%$ and $83.48 \%$ ) of the RDT and UMT respectively. There was also a similarity in the different diagnostic characteristics of both techniques. These findings were similar to the reports by Tagbo Oguonu et al, in 
2014 [17]. This indicates that the UMT could aid in the clinical management of suspected malaria cases in our setting.

Our study demonstrated a high prevalence of malaria (46.50\%), which was a little higher than the $20 \%$ reported by Tagbo et al. This discrepancy could be attributed to differences in study site and also our study involved only febrile symptomatic patients while Tagbo's study considered asymptomatic participants. The pre-test probability of disease in a patient who tests positive by the UMT is $45.5 \%$ while that for RDT was $44 \%$. For example, upon receiving a negative UMT result for a suspected malaria case, the clinician now knows that this patient's probability of having malaria parasites detectable by microscopy is unlikely, only $4 \%$. In contrast, a positive UMT result would indicate that the probability of detecting malaria parasites in this patient by microscopy is $46 \%$.

Hence, the UMT could potentially expand malaria testing in the health care settings, particularly in hard-toreach locations or health care facilities where blood draw is difficult or impractical for microscopy, and advance the current global effort toward universal diagnosis in cases of fever suspected of being malaria.

The high degree of sensitivity from the population from 0-20 years of age, may suggest that the UMT is able to detect acceptable level of antigens especially in areas of high malaria transmission. Also the false positivity related to the presence of the gametocyte is indicative of the ability to detect sexual form of $P$. falciparum a factor which is useful in absolute sensitivity tests against the clinical episodes that was used in this study. However, in areas of low malaria endemicity, this level of false positives may create drug wastage, which the current malaria control efforts seek to reduce. False positive results may be attributed to the ability of all histidine-rich protein 2 (HRP2) antigen malaria test kits to detect the parasite antigen even after the malaria illness. The presence of rheumatoid factor and schistosomiasis in a patient may also lead to false positivity, and will need to be further evaluated [19]. These factors are known to affect the blood type malaria RDTs, but little is known about such influence on the urine malaria test kits. It may be assumed that since both (blood and urine-based) test kits are specific for HRP2 such effect may also occur with the UMT.

The false negative results that were gotten from UMT are comparable to those of blood-specific malaria RDTs. Many factors have been described to contribute to the false negative results with HRP2-based rapid test kits. These include parasite and host factors such as deletion or mutation of HPR2 gene and an illustration of the prozone effect observed with immunochromatographic tests such as malaria RDT [19, 20].

Some of the limitations observed with the use of the UMT was the delay in provision of urine by some subjects, particularly among children. This may be a delay factor in the promptness of testing and treatment. Again, the prevalence of malaria for our study was limited to a single plasmodia species; $P$. falciparum, hence not revealing the true prevalence of malaria in the community as there could be infections with other plasmodium species.

\section{CONCLUSION}

The Urine Malaria Test kit that was evaluated in comparison to the blood based RDT, showed a lot of similarities with blood smear microscopy as gold standard. Hence, it can be used in our setting for the prompt and accurate diagnosis of malaria in febrile patients. 


\section{ABBREVIATIONS.}

WHO: World Health Organization.

PCR: Polymerase Chain Reaction

PfHRP2: P. falciparum Histidine-Rich Protein 2

RDTs: Rapid Diagnostic Tests

UMT: Urine Based Malaria Test Kit

P. f: Plasmodium falciparum

PLR: Positive Likelihood Ratios.

PPV: Positive Predictive Value

NPV: Negative Predictive Value

NLR: Negative Likelihood Ratios

\section{ACKNOWLEDGEMENT}

The authors wish to thank the Directors of the Buea and Limbe Regional Hospitals, the staff of the Laboratories of these hospitals. Our sincere gratitude also goes to the participants who willfully took part in the study.

\section{FUNDING}

This study was self-funded but a substantial financial support came from the Adolphe Monkiedje Fellowship Award.

\section{AVAILABILITY OF DATA AND MATERIALS}

All the data supporting our findings have been presented in this paper.

\section{AUTHORS' CONTRIBUTIONS}

BRN conceived, designed, and coordinated the study, took part in data collection, analyses and interpretations, conducted the literature search and review, and co-wrote the paper. ACAN and NJCA designed and supervised the study, participated in the statistical analyses, and critically revised the paper. ADB participated in the data collection, analyses and critically revised the paper.

\section{COMPETING INTERESTS}

The authors declare that they have no competing interests.

\section{CONTRIBUTORS INFORMATION.}

Achu Che Awah Nforbegwe. Email: niawah95@gmail.com

Bihnwi Rengerline Nchotu. Email: rbihnwi@gmail.com

Agnes Djema Bongah. Email: bongahdjema@yahoo.com

Nguedia Jules Clement Assob. Email: juleclement@yahoo.fr 


\section{REFERENCES.}

1. World Health Organization. 2014. World malaria report 2014. World Health Organization, Geneva, Switzerland.

2. WHO. World Malaria Report. Geneva: World Health Organization; 2012.

3. Sowunmi A., AkindeleJ, A. Presumptive diagnosis of malaria in infants in an endemic area. Trans R Soc Trop Med Hyg 2011, 87: 422.

4. Nevill C.G. Malaria in sub-Saharan Africa. SocSci Med, 2010 31: 667-669.

5. Reyburn H.,Mbakilwa, H., Mwangi R.,Mwerinde O.,Olomi, R.,Drakeley C., and Whitty C.J. Rapid diagnostic tests compared with malaria microscopy for guiding outpatient treatment of febrile illness in Tanzania: randomised trial. Brit Med Jour, 2010. 7590:334403. 
6. Rafael M.E., Taylor T.,Magil A., Lim Y.W.,Girosi F., and Allan R. Reducing the burden of childhood malaria in Africa: the role of improved. Nat, 2011, 444:39-48.

7. Achary K.G.,JavedAkhtar S. D., Monalisa R., Shikha S. "A Non Invasive Approach for Rapid Malaria Diagnosis from Urine and Saliva of Malaria Patients" Volume II, Issue III, March 2015 IJRSI ISSN 2321 - 2705

8. Wellington A.O., Nnenna E., Godwin N., Oladipo O.O., Kaitlin. Wendy O', Evaezi O., and William B: Multicenter Pivotal Clinical Trial of Urine Malaria Test for Rapid Diagnosis of Plasmodium falciparum Malaria. J of clinmicrobiol 2017 vol. 55 no.53-263.

9. Kwenti ET, Nkume FA, Tanjeko AT, Kwenti TDB. The effect of intestinal parasitic infection on the clinical outcome of malaria in coinfected children in Cameroon. PLoS Negl Trop Dis. 2016;10 (4):e0004673. doi:10.1371/journal.pntd.0004673

10. Wanji S, Kengne-Ouafo AJ, Eyong EJ, Kimbi HK, Tendongfor N, Ndamukong-Nyanga $\mathrm{JL}$, et al. Genetic diversity of Plasmodium falciparum merozoite surface protein-1 block 2 in sites of contrasting altitudes and malaria endemicities in the Mount Cameroon region. Am J Trop Med Hyg. 2012; 86:764-774. doi: 10.4269/ajtmh.2012.11-0433.

11. Bigoga JD, Manga L, Titanji VPK, Coetzee M, Leke RGF. Malaria vectors and transmission dynamics in coastal south-western Cameroon. Malar J. 2007;6:5. doi: 10.1186/1475-2875-6-5.

12. Njunda AL, Assob NJC, Nsagha SD, Kamga FHL, Mokenyu MD, Kwenti ET. Comparison of capillary and venous blood using blood film microscopy in the detection of malaria parasites: A hospital based study. Sci J Microbiol. 2013;2(5):89-94.

13. Moll K., Ljungstrom I., Perlmann, H., Scherf A., Wahlgren M. Methods in Malaria Research. Malaria Research and Reference Reagent Resource Center (MR4), Manassas Virginia:2010. MR4/ATTC, Google Scholar.

14. Maltha J, Gillet P, Bottieau E, Cnops L, van Esbroeck M, Jacobs J. Evaluation of a rapid diagnostic test (CareStart ${ }^{\mathrm{TM}}$ Malaria HRP-2/pLDH (Pf/pan) Combo Test) for the diagnosis of malaria in a reference setting. Malaria J. 2010;9:171. doi: 10.1186/14752875-9-171. [PMC free article] [PubMed] [Cross Ref]

15. https://www.google.com/Fyodor+urine+malaria+test accessed on August 9, 2017.

16. Coleman R.E., Sattabongkot J.,Promstaporm S.,Maneechai N.,Tippayachai B., Kengluecha A., Rachapaew N., Zollner G., Miller R.S., Vaughan J.A., Thimasarn K., and Khuntirat B. Comparison of PCR and microscopy for the detection ofasymptomaticmalaria in a Plasmodium falciparum/vivaxendemic area in Thailand.Mal J2009, 5:121-127. 
17. Tagbo O., Elvis S., Bertilla U. E., BaoLige A., Rich E.U., and Eddy A. "The performance evaluation of a urine malaria test (UMT) kit for the diagnosis of malaria in individuals with fever in south-east Nigeria: cross-sectional analytical study" Mal J 2014, 13:403

18. Nwakanma D.C., Gomez-Escobar N., Walther M., Crozier S., Dubovsky F., Malkin E., Locke E., Conway D. J. Quantitative detection of Plasmodium DNA in saliva, blood and urine. J Infect Dis 2009, 199:1967-1974.

19. Rodriguez-del-Valle M., Quakyi I.A., Amuesi J., Quaye J.T., Nkrumah F., Taylor D.W. Detection of antigen and antibodies in urine of humans with Plasmodium falciparum malaria. J ClinMicrobiol 2011.29:1236-1242.

20. Murray C.K., Bennett J.W. Rapid diagnosis of malaria. Interdiscip Perspect Infect Dis 2009, 2009:415953. doi: 10.1155/2009/415953. 


\section{Supplementary Files}

This is a list of supplementary files associated with this preprint. Click to download.

- Tables.pdf 